\title{
18 Nogaier als Faktor der frühneuzeitlichen Krim-Geschichte
}

\begin{abstract}
[L]eur obéissance est fort privilégiée. Ils le [le Chan] suivent á la guerre, lui donnent une partie de leur butin, et lui payant pour chaque prisonnier depuis un rixdaler [= Reichstaler] jusqu'á trois ducates [...]. Ils mangeant de la chair de cheval, buvant le lait de jument, en un mot ayant aussi peu de goût pour la propreté que leurs ancêtres, ils ont le visage plat, d'un brun-noirâtre, et ridé, les yeux petits et enfoncés, le nez recourbé en dedans et peu de barbe. Ils sont enclins au pillage, et quand ils peuvent trouver l'occasion de dépouiller quelque voyageur, ils ne la manquent pas; mais ils ne sont pas meurtriers, et des qu'ils se croient en sureté, ils préfèrent de vendre leur prisonniers. ${ }^{1}$
\end{abstract}

Diese 1784 erstmalig (und damit posthum) veröffentlichten Zeilen hatte der schwedische Gelehrte Hans Erich Johann Thunmann (1746-1778) verfasst. Der an der Universität in Halle/Saale wirkende Skandinavist, Balkanforscher und Romanist bekleidete einen Lehrstuhl mit der beeindruckenden Denomination „Professur für Weltweisheit und Beredsamkeit.“2 Mit dem östlichen Europa einschließlich des Schwarzmeerraumes hatte er sich nicht nur in seiner kleinen Abhandlung über den „krimschen Staat“, wie der Titel im deutschen Original lautete, befasst, sondern auch in dem umfänglichen Werk „Untersuchungen über die Geschichte der östlichen europäischen Völker.“3 Wie in der Beschreibung des Dominikanermönchs de Luc über die Krimtataren wird auch hier von Kriegern berichtet, die offenbar eine symbiotische Beziehung zu Pferden hatten, Pferdefleisch („la chair de cheval“) aßen und Stutenmilch (,le lait de jument“) tranken und damit wie ihre Vorfahren wenig Geschmack gehabt hätten. Auch sie machten Gefangene, durch Raubzüge oder - und hier beginnt der Bericht des schwedischen Wissenschaftlers von dem de Lucs zu differieren - durch den Überfall auf Reisende: Diese wurden bei Gelegenheit beraubt, aber - denn „sie sind keine Mörder“ - nicht umgebracht, da es besser gewesen sei, sie zu verkaufen. Alsdann berichtet Thunmann recht ausführlich vom Aussehen der von ihm Beschriebenen. Diese entsprachen ganz offenbar nicht dem ihm bekannten ,europäischen Typ', sahen aber auch nicht wie die typischen Krimtataren der Südküste oder der

1 Thounmann (1786), 63f. Das deutsche Original Thunmann (1784) lag nicht vor.

2 J Eberhard (1779).

3 Thunman (1774). Darin ging es um die „Allgemeine Geschichte der Völker welche an dem Schwarzen Meere und der Mäotischen See bis auf den Einfall der Mogolen [sic], gewohnt haben.“ In dem Kapitel „Über einige Gegenstände der russischen Geschichte“ befasste er sich u.a. eingehend mit dem Anteil aus Skandinavien stammender Ethnien an der Geschichte der Kiewer Rus’ und positionierte sich in der sog. Warägerfrage bzw. Normannentheorie, 367-406, hier besonders 371-390.

Ә OpenAccess. ( 2020 Kerstin S. Jobst, publiziert von De Gruyter. (cc))BY Dieses Werk ist lizenziert unter der Creative Commons Attribution 4.0 International. https://doi.org/10.1515/9783110520620-020 
Bergregionen aus, so steht zu vermuten. Der Professor für „Weltweisheit und Beredsamkeit“ schilderte vielmehr Menschen (wieder einmal Männer), die deutlich anders aussahen mit ihren flachen, dunklen und faltigen Gesichtern sowie gebogenen Nasen: die Nogaier. Bereits de Luc hatte phänotypisch einen Unterschied zwischen den Tataren der Steppe und des Südens ausgemacht und damit eine Charakteristik vorgelegt, die sich in den nächsten Jahrhunderten als recht stabil erweisen sollte ${ }^{4}$ : Die Nogaier hätten ein flaches und volles Gesicht, einen großen Kopf, kleine Augen und eine platte Nase. Er vergegenwärtigte immerhin, dass es auch Nogaierinnen gab, die allerdings nur in der Jugend hübsch seien, im Alter hingegen hässlich, so sein Urteil. ${ }^{5}$

Unstrittig ist, dass sich die Ethnogenese der Nogaierinnen und Nogaier linguistisch, kulturell und anthropologisch von der der Tatarinnen und Tataren in den Bergregionen und an der Küste unterschied und die Ähnlichkeit mit den BewohnerInnen der kasachischen Steppe viel größer war. ${ }^{6}$ Dies trifft auch auf die Lebensweise zu, lebten die in den nördlichen Steppen der Krim und des angrenzenden Festlandes siedelnden NogaierInnen doch bis weit in das 19. Jahrhundert zumindest teilweise nomadisch; anders hingegen die übrigen TatarInnen der Krim, die weitaus früher in einen wechselseitigen Akkulturationsprozess mit den Nachfahren der griechischen, gotischen, alanischen, italienischen oder anderer BewohnerInnen eingetreten waren, ganzjährig in Städten oder Dörfern wohnten und sich vorrangig mit Handel, Handwerk oder mit Wein- ${ }^{7}$, Tabak- oder Obstanbau befassten. Beide Gruppen standen dennoch in einem engen, auch wirtschaftlichen, Austauschverhältnis, denn ,der Süden“ war vom ,Norden“ in Bezug auf in der Steppe produzierte Waren aus der Weidewirtschaft abhängig. Umgekehrt benötigte der ,Norden` den ,Süden“ als Absatzmarkt und in dessen Funktion als Zwischenhändler. Ökonomie und Lebenswelten hatten auch Einfluss auf die Religionsausübung, folgten sesshafte TatarInnen doch eher dem osmanischen Vorbild islamischer Praktiken, die insgesamt institutionalisierter waren als die der nomadischen Gruppen. ${ }^{8}$ Dies wurde von nicht-muslimischen AutorInnen häufig dahingehend interpretiert, dass die Nogaier weniger gläubig, weniger ,fanatisch' seien. ${ }^{9}$ Interessant ist in diesem Zusammenhang, dass das als fremd wahrgenommene Aussehen und die überwiegend nomadische Lebens-

\footnotetext{
4 Vgl. dazu Jobst (2007b), 90 f., $156 \mathrm{f}$.

5 Opisanie (1879), 487.

6 Dazu ausführlicher Williams (2001), 88f., dem ich hier folge.

7 Auch in Zeiten des Krim-Chanats wurde auf der Halbinsel Wein produziert und konsumiert, auch von den muslimischen Krimtataren. Vgl. dazu Halenko (2004), 507-547.

8 Vgl. Williams (2001), $58 \mathrm{f}$.

9 Jobst (2007b), u. a. 220.
} 
weise der Nogaier die sesshaften europäischen AutorInnen dennoch häufig urteilen ließ, dass die übrige tatarische Bevölkerung der Krim zivilisierter und damit attraktiver sei. So schwärmte beispielsweise ein russischer Reisender der zweiten Hälfte des 19. Jahrhunderts von den Bergtatarinnen, die helle Haut hätten, europäisch aussähen und somit „manchmal sogar schön“ seien. Dies gelte auch für die kleinen Tataren und Tatarinnen, die den russischen Kindern ähnlich seien, da in ihnen kein mongolisches Blut fließe. Der Autor schloss:

Wenn Du Dich an viele Sitten der Tataren des südlichen Ufers erinnerst, die Freiheit ihrer Frauen, die Achtung einiger christlicher Feiertage und Denkmäler, ihre Liebe zur Sesshaftigkeit, und diese Befunde mit ihrer äußeren Erscheinung vergleichst, so bist Du auf den ersten Blick davon überzeugt, dass die sog. Tataren dem kaukasischen Stamm ebenso nahe sind wie wir selbst. ${ }^{10}$

Vergleichbare Hierarchien zwischen ,uns' und dem ,Fremden' in seinen konstruierten Abstufungen entwickelten viele europäische AutorInnen, wie z.B. der in seiner Zeit recht populäre Entdecker und Reiseautor Harry Willes Darell de Windt (1856-1933). Er hielt die Küstentataren für „more refined in appearance and manner" als die Nogaier und führte dies auf die Verbindungen mit den Griechen und Genuesen zurück. Zugleich seien sie aber träger („more indolent“), was de Windt für eine Folge des milden südlichen Klimas hielt. ${ }^{11}$

Die Nogaier Horde war eines der der Goldenen Horde nachfolgenden Herrschaftskonglomerate und führte ihren Namen auf den Emir und erfolgreichen mongolischen Feldherren Nogai (?-1299) zurück. Wie andere Horden waren die Nogaier ethnisch heterogen und in Clans organisiert. Vom 15. Jahrhundert an drangen sie von Osten her weit in die Pontisch-Kaspischen Steppen vor, zeigten sich in ihrer Bündnispolitik sehr geschmeidig - und traten nach den eindrucksvollen Erfolgen des Moskauer Reiches gegenüber den Chanaten von Kazan' und Astrachan' im 16. Jahrhundert zu ihrem Schutz in ein informelles Suzeränitätsverhältnis mit dem Krim-Chanat. ${ }^{12}$ Wie das Krim-Chanat selbst gegenüber dem Osmanischen Reich, so zeichneten sich auch die Nogaier als Vasallen durch Eigensinn aus. Keinesfalls folgten sie regelhaft der Politik Bağçasarays gegenüber der Steppe, zogen u.a. selbständig in den Norden, was sich eben auch in der eigenmächtigen Beschaffung von SklavInnen (s.o.) zeigte. Ihre Raubzüge trugen ebenfalls dazu bei, dass slavische SiedlerInnen die nördlichen Steppengebiete der

10 Markov (1994), 212. Dies ist ein beredtes Beispiel für die Anwendbarkeit des Ähnlichkeitskonzepts, das auch in kolonialen Kontexten eine Rolle spielt. Vgl. Bhatti u. Kimmich (2015).

11 Windt (1917), 187.

12 Fisher (1978), 24. 
Krim nicht dauerhaft und nur in geringer Zahl bewohnen konnten. Bis in die unmittelbare Zeit vor der Herauslösung des Krim-Chanats aus der osmanischen Einflusssphäre in der zweiten Hälfte des 18. Jahrhunderts blieben die Nogaier ein aufrührerisches Element innerhalb des Krim-Chanats und ließen sich phasenweise von dessen Gegnern instrumentalisieren. ${ }^{13}$

13 Fisher (1977), 69f. 